\title{
ORIGIN OF ECHOES IN THE ECHOENCEPHALOGRAM
}

BY

\section{BRITA LITHANDER}

\author{
From the Paediatric Clinic, Karolinska Sjukhuset and the Neurological Clinic, Södersjukhuset, Stockholm, Sweden
}

Echoencephalography was described in 1956 by Leksell, who showed that the ultrasonic echo "could be used for practical diagnostic purposes through the intact skull'. According to Leksell, when recording at the temporal region with the probe directed toward the corresponding region on the opposite side of the skull, an echo regularly appears which derives from the midline structure of the brain. Leksell's method has proved very useful, since it is quick, simple, and relatively reliable. In addition to the midline echo, Leksell observed a number of other echoes, "which appear to come from the boundaries between brain tissue and cerebrospinal fluid'.

In a later publication, Leksell (1958) expressed the opinion that the pineal body is the prime source of the midline echo. Vlieger and Ridder (1959) found that the midline echo originates in the longitudinal fissure, not in the falx cerebri. They also recorded echoes which they believed derived from the ventricles. Gordon (1959) expressed the opinion that the midline echo originates primarily in the septum pellucidum and also in the third ventricle, and that these echoes are often indistinguishable.

The investigations made hitherto have not provided a satisfactory answer to the question whether one or more structures yield the midline echo. Nor has the origin of the lateral echoes been fully clarified, and further study is required to elucidate this problem.

The purpose of the present study is to try to determine from what structures in the brain the midline echo and the lateral echo derive.

To locate the structures from which echoes originate, I have developed a method using hypodermic needles as indicators, and a water tank method has been evolved in order to localize these structures with greater precision.

\section{Physical Principles}

Ultrasound is produced when short pulses of highfrequency electrical energy are applied by a transmitter to a piezoelectric crystal which produces the mechanical vibrations. Ultrasound is propagated at different velocities in different media. An interface between two media with different velocities of propagation of ultrasound reflects part of the ultrasound back to the crystal where it is retransformed to electric energy. Air is a poor medium for propagation of ultrasound with the ultrasound frequencies used in this investigation, practically all the ultrasound being reflected by an interface of air.

The reflection of ultrasound in general follows the laws of optics. Consequently, if the ultrasound meets an interface between two media at right angles, some of the sound passes through and some will be reflected back to the crystal; with a convex surface the reflected beam will diverge, and with a concave surface it will converge.

In water the ultrasound at first is propagated without diverging over a distance which varies with the frequency? and with the diameter of the probe. Thereafter, theo sound beam diverges with a specific angle of deviation $\mathbb{D}$ With a $2 \mathrm{Mc} / \mathrm{sec} .10 \mathrm{~mm}$. probe, the length of the parallel beam is $34 \mathrm{~mm}$., and with a $2 \mathrm{Mc}$. $/ \mathrm{sec} .24 \mathrm{~mm}$. probe it is $215 \mathrm{~mm}$.

\section{Apparatus}

The standard ultrasonic echo apparatus (Krautkrämer model USIP 9) was used with an adapted Polaroid-Land camera (Leksell, 1958).

The following types of barium titanate probes were used: $2 \mathrm{Mc}$. $/ \mathrm{sec}$. $24 \mathrm{~mm}$. diameter for examinations through the intact skull in adults; $2 \mathrm{Mc} / \mathrm{sec} .10 \mathrm{~mm}$. diameter for direct application on the brain in adults; $4 \mathrm{Mc}$. $/ \mathrm{sec}$. $10 \mathrm{~mm}$. diameter or $2 \mathrm{Mc}$. $/ \mathrm{sec}$. $10 \mathrm{~mm}$. for examinations in infants. Liquid paraffin was used as a coupling medium between probe and skull.

\section{Method I}

Determination of Extent of Midline Echoes in Infants. -In adults the midline echo can usually be recorded only in a small area in the temporal region. This may be because the echo-producing midline structure has sug a limited extent in the sagittal plane of the skull that it can only be recorded at right angles to the sagittal plane from a very small area. It may also be due to the fact that the skull is thinner or less dense here and that ultrasound therefore passes through more easily. A study of the midline echo in infants, whose entire skull is thin, was therefore considered useful.

The investigation was made on nine healthy newborn infants and two infants who had died from asphyxia at 


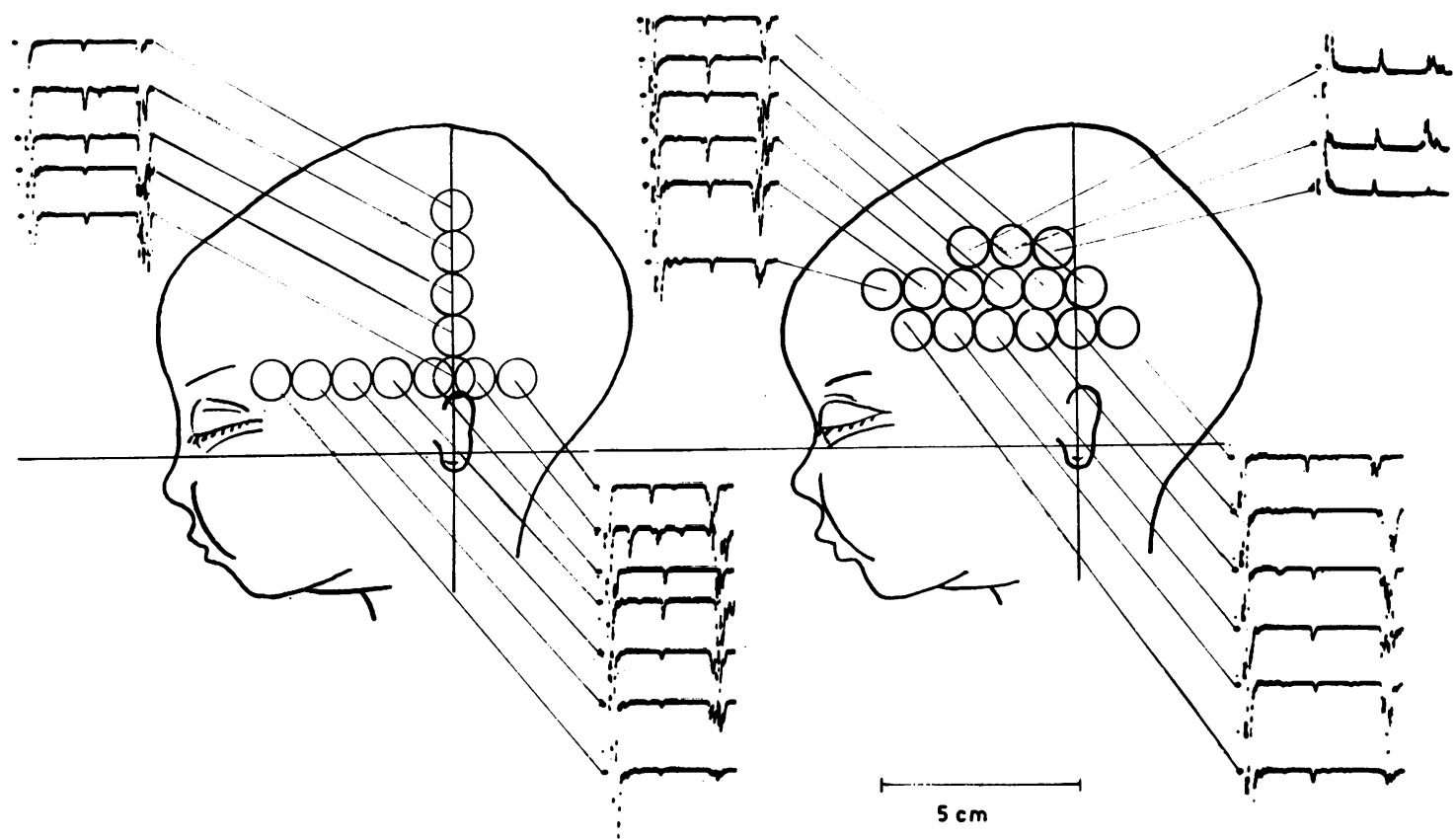

Fig. 1.-Extent of midline echoes in infants.

birth. No difference in the echoencephalograms was found between the living and the dead infants.

To secure corresponding measuring points from case to case, I used a system of coordinates; the abscissa is formed by an imaginary baseline through the external auditory meatus and the lower edge of the orbit; the ordinate is a line through the external auditory meatus at an angle of $90^{\circ}$ to the baseline, the aural ordinate.

\section{Results}

With the probe $(2 \mathrm{Mc}$. $/ \mathrm{sec} .10 \mathrm{~mm}$.) placed $1 \mathrm{~cm}$. above the baseline and applied at right angles to the mid-sagittal plane, a distinct midline echo was recorded along a horizontal line extending approximately 4 to $5 \mathrm{~cm}$. in front of and 1 to $2 \mathrm{~cm}$. behind the aural ordinate. The midline echo was also recorded to about the same extent along a line 2 or $3 \mathrm{~cm}$. above the baseline. Recording along the aural ordinate gave a distinct midline echo for a distance of 4 to $5 \mathrm{~cm}$. (Fig. 1). The midline echo in infants could thus be recorded over large areas of the frontal, temporal, and parietal regions, i.e., within the entire area in which the probe could be applied almost at right angles to the sagittal plane of the skull.

In children whose skulls are flattened laterally this area is larger than in those with more spherical heads. Under favourable conditions, the midline echo can thus be recorded over a relatively large area. This indicates that the midline echo in children is not solely produced by a small structure like the pineal body. It might be concluded from these observations that probably the most important cause of the limitation of the area from which the midline echo derives in adults is the density which characterizes the adult skull, with the exception of the relatively thin temporal bone.

\section{Method IIA}

Location of Echo-producing Structures with Hypodermic Needles.-The roof of the skull of a cadaver is sawed off and carefully removed. To prevent leakage of the cerebrospinal fluid it is important not to damage the cerebral meninges.

Echoencephalography is done before and after removal of the roof of the skull to check that air has not entered the blood vessels and cavities of the brain and thus produced echoes not normally recorded in vivo.

A $2 \mathrm{Mc}$. $/ \mathrm{sec} .10 \mathrm{~mm}$. probe can be used when recording directly on the brain. This is of great advantage because this probe gives a narrower beam and has a greater resolving power than the larger $2 \mathrm{Mc}$. $/ \mathrm{sec} .24 \mathrm{~mm}$. probe. Furthermore, the initial pulse is shorter and thus scarcely encroaches on the echoencephalogram.

The probe is placed on the temporal lobe of the brain, a midline echo is sought and the probe is adjusted accordingly.

A needle inserted into the brain at right angles to the ultrasonic beam gives a strong echo, and this fact can be utilized in locating an echo-producing structure. A long needle is inserted in the brain and is moved until its echo coincides with the echo to be analysed. The needle is inserted to a depth at which its echo is barely visible on 


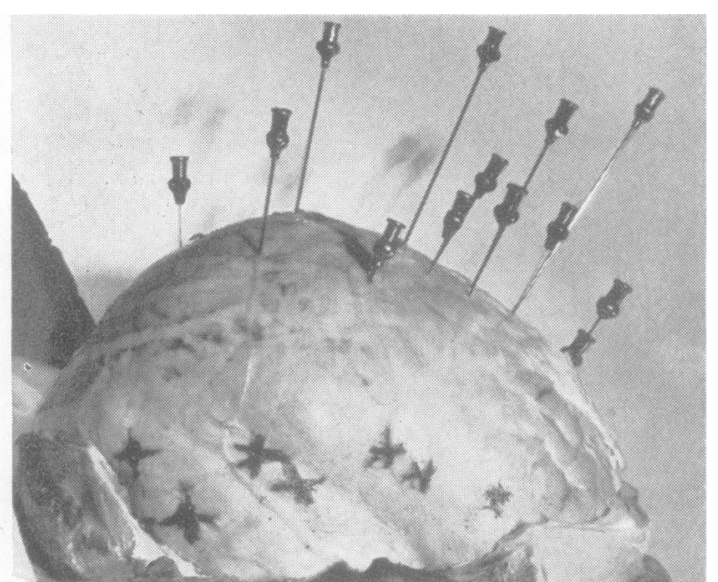

FIG. 2.-Brain with needle indicators inserted. Crosses show positions of the probe at the various recordings.

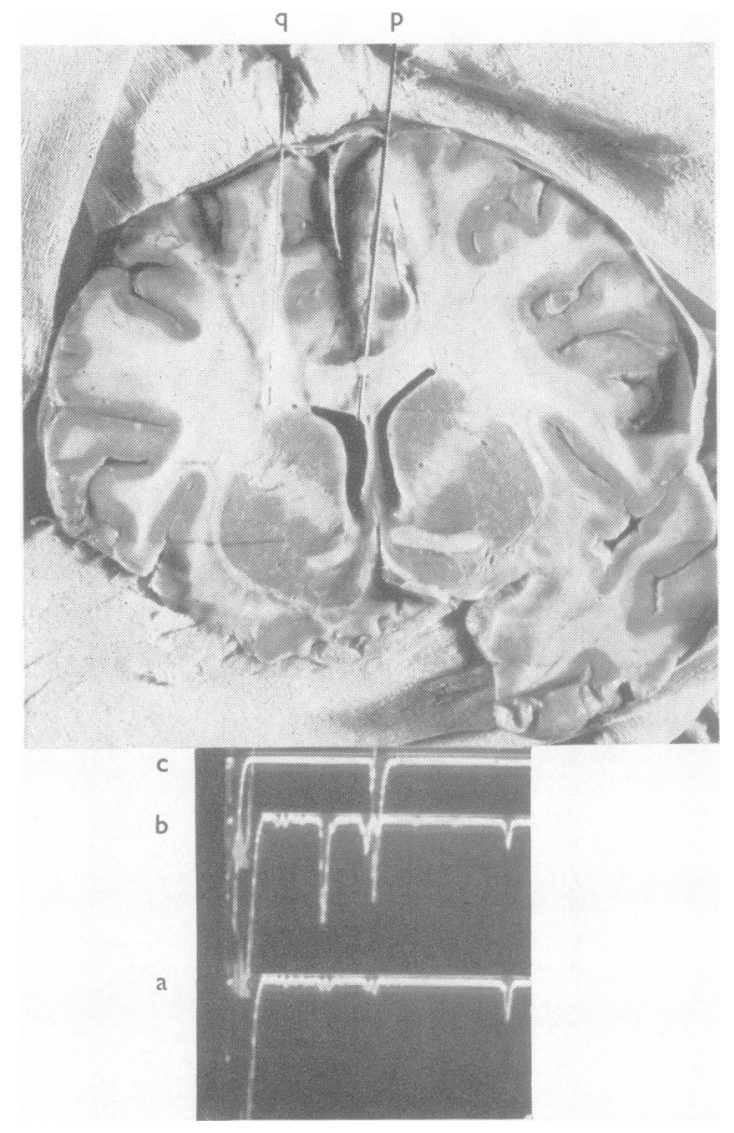

FIG. 3-Method IIA. Section of brain with points of needles near the left lateral ventricle and close to the septum pellucidum. Recording from the left side directly on the brain: a, echoencephalogram before insertion of needles; $b$, echoencephalogram after insertion of needles $\mathrm{p}$ and $\mathrm{q}$; $\mathrm{c}$, control. the echoencephalogram, and is then left in that position (Fig. 2). Formalin is then injected into the carotid arteries and the brain is removed and sectioned. The location of the needle can now be determined and conclusions can be drawn concerning the structure from which the echo emanates.

\section{Results}

Seven cases were studied with method IIA and 59 echoes were analysed. In order to illustrate the results of the investigations many figures have been included, and have been selected with the object of illustrating the different structures from which an echo can be recorded. The same structures have yielded echoes in different patients, with the exception that in some instances it has not been possible with certainty to relate the echo to a definite anatomical structure.

Record a in Fig. 3 shows the appearance of the echoencephalogram before needles were inserted into the brain. We see the initial echo at the extreme left; at the extreme right is the bottom echo from the opposite surface of the brain. Between these two echoes, two double echoes are recorded; the one on the right is the midline record $b$, and was $N$ made when two needles were moved until theif 을 respective echoes coincided precisely on the echo $\triangle$ encephalogram with the first spike in each double 7 echo. Record c is the 'control deflection', which o indicates the sagittal midplane (Lithander, 1960). This g is the same as the transmission pulse described be Vlieger and Ridder (1959). This method of ascerô $\overrightarrow{0}$ taining the exact midpoint has also been used by Jeppson (1960).

We see in the picture that the left needle appears to point to the left ventricle, although the latter was accidentally cut somewhat obliquely. The right needle points to the left side of the septum pellucidum. The second spike in the right double echo therefore presumably represents the right side of the septum pellucidum. This is in agreement with the location of the control echo between these echoes, which derive from the left and right side of the septum pellucidum.

Fig. 4 shows, likewise, the position of the needles in the Sylvian fissure and in the third ventricle. Fig. 5 illustrates how the echoes in record a are identified by the needles. The right needle is inserted behind the others and can be seen protruding from the brain. Its point is, however, in the bottom of the lateral ventricle at the boundary between brain and cerebrospinal fluid. The next two needles appear to be situated on the boundary between gray and white substance. The last needle is in the Sylvian fissure. Fig. 6 shows echoes which originate from the lateral ventricle and the longitudinal fissure or from the falx. 


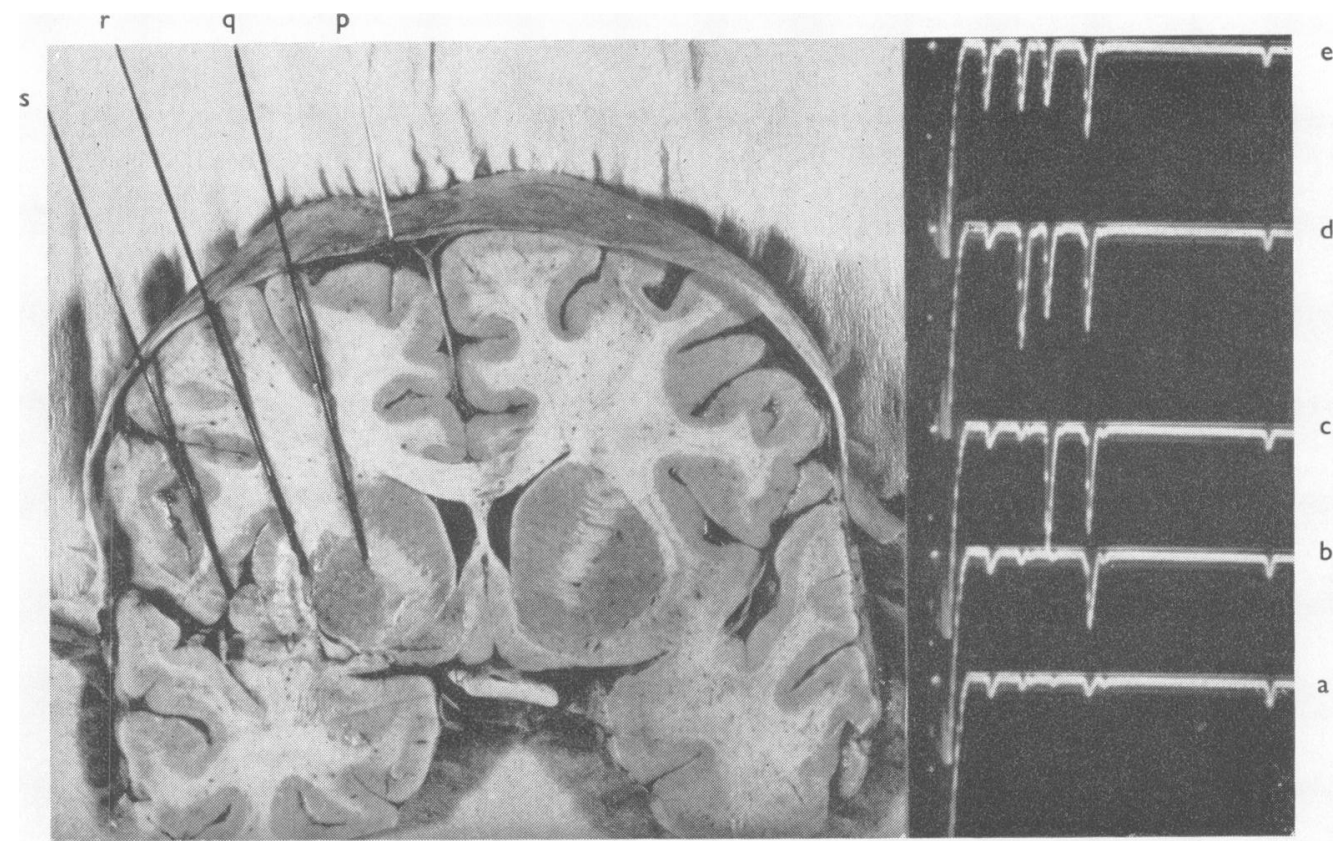

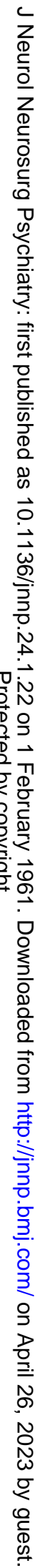

FIG. 5.-Method IIA. Section of brain with points of needles in the Sylvian fissure in the boundary between white and gray matter and in the left ventricle. Recording from the left side: $a$, before insertion of needles; $b$, after insertion of needle p; c, after insertion of needles $p, q$; , after insertion of needles $p, q, r ; e$, after insertion of needles $p, q, r, s$.

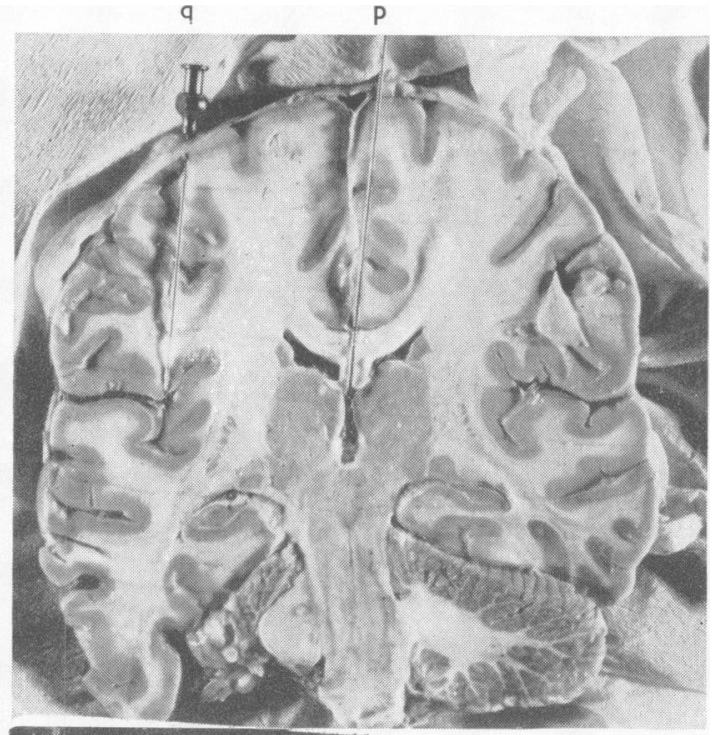

d

c

b

a


q; d, control.

\begin{abstract}
b, aft needle $\mathrm{p}$; $\mathrm{c}$, after insertion of needles $p$ Section of brain with points of needles in in the third ventricle. Recording from the left side: a, before
\end{abstract}

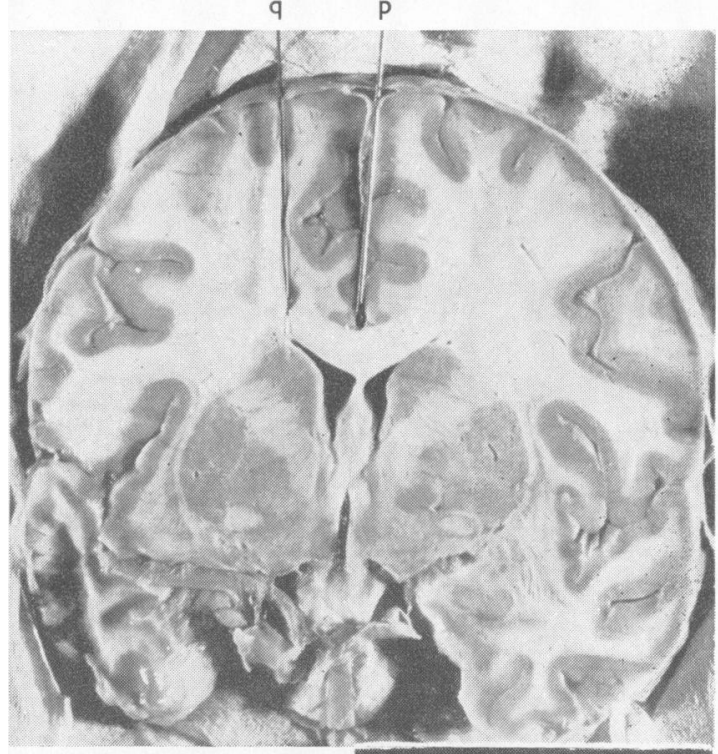

FIG. 6.-Method IIA. Section of brain with points of needles in the left lateral ventricle and in the longitudinal fissure. Recording from the left side: a, before insertion of needles; b, after insertion of needles p, q; c, control.

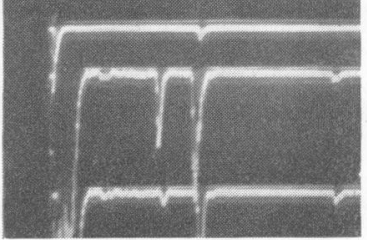


With this method, the sagittal plane in which the echo-reflecting structure is situated can be determined. Owing to the width of the ultrasonic beam, however, it is not possible to determine exactly in which horizontal or frontal plane this structure is located. But in the present investigation, the position of the point of the needle corresponds to the upper limit of the beam. The structure from which the echo derives is thus to be found in the same horizontal plane as the point of the needle or below it.

In many cases, however, there is no difficulty in determining the structure from which a specific echo derives. In Fig. 3, for example, the right needle points to the septum pellucidum, and there is scarcely any doubt that the midline echo derives from the septum pellucidum. The left needle, on the other hand, is not in exactly the same frontal plane as the horn of the lateral ventricle. A comparison with the right lateral ventricle shows that the position of the point of the needle in the sagittal plane corresponds to the lateral horn of the left lateral ventricle.

Using this method, we thus find that the midline echo derives from a number of different structures in the midline. The lateral echoes come from the boundary between brain substance and cerebrospinal fluid (ventricles and gyri) and between gray and white substance (thalamus, internal capsule, and external capsule).

It should be borne in mind, however, that conditions are more favourable when examinations are made on the brain itself than through the intact skull, and that more echoes can be recorded than when the ultrasound has to pass through the skull.

\section{Method IIB}

Method II was again used but a probe was placed on the skull instead of directly on the brain in order to make the experimental conditions resemble a routine clinical examination as closely as possible. A strip of bone measuring approximately 1 to $2 \times 10 \mathrm{~cm}$. is sawed out of the roof of the skull and a probe $(2 \mathrm{Mc}$./sec. $24 \mathrm{~mm}$.) is placed on different points over the temporal region. The midline echo is sought at these points, and a needle is inserted into the brain through the opening in the skull. As in the foregoing investigation, the needle echo is made to coincide with the echo to be analysed. Formalin is then injected into the carotids and the brain is removed and sectioned.

\section{Results}

Fig. 7 shows the needle in the septum pellucidum. In this case it is impossible to determine whether the midline echo derives from the third ventricle or the septum pellucidum.

From Fig. 8 it is evident that the needle penetrates the midplane of the pineal body down toward the aqueduct. Considering that the point of the needle is situated in the upper horizontal plane of the ultrasonic beam, the echo presumably derives from the aqueduct.

Fig. 9 shows a double midline echo and the needle (in this special case inserted slightly too deep) indicates that the echo derives from the third ventricle.

Fig. 10 shows a lateral echo produced by the lateral wall of the posterior horn. The midline echo appears to be derived either from the longitudinal fissure or the falx. The boundary surfaces between the longitudinal fissure and the falx are so close to one another that it is impossible to determine with a needle in which of the two an echo arises. If the falx was touched with the needle, the echo changed its appearance, which was not the case if the inner surfaces of the hemispheres were touched.

Fig. 11 illustrates an attempt to determine if it is really the falx that yields the midline echo. In this experiment, the falx was cut in two rostrally to the probe through the opening in the roof of the skull. When this was done, the midline echo disappeared, but it recurred every time the falx was stretched (Fig. 11a, b, c).

No midline echo could be recorded if the falx was removed by drawing it occipitally to the probe (Fig. 11d). It reappeared when the falx was again drawn forward in a rostral direction and stretched (Fig. 11e). The falx thus yields a distinct echo.

This investigation with the probe placed on the outer surface of the skull reveals, like recordings directly on the brain, that the midline echo arises from different structures in the midline and that the interface between brain tissue and cerebrospinal fluid yields an echo. The width of the ultrasonic beam makes it difficult to determine whether the echo from the boundary between gray and white substance is recorded on the skull; however, a number of recordings indicated that this is the case.

With this method, it is possible to determine the location of the echo-producing structure in the sagittal plane only. Sometimes this is sufficient to identify a specific echo, but sometimes one may also need to determine in which horizontal and vertical plane the echo arises, and the investigations were therefore continued with another method to meet this situation.

\section{Method III}

Echoencephalography with Brains Immersed in a Watertank.-A probe $(2 \mathrm{Mc}$. $/ \mathrm{sec}$. $24 \mathrm{~mm}$.) is applied to the outside of a plastic tank filled with degassed water in such a way that half the surface of the probe is above the level of the water. A bottom segment of the surface of the probe is screened off with a double layer of plastic 

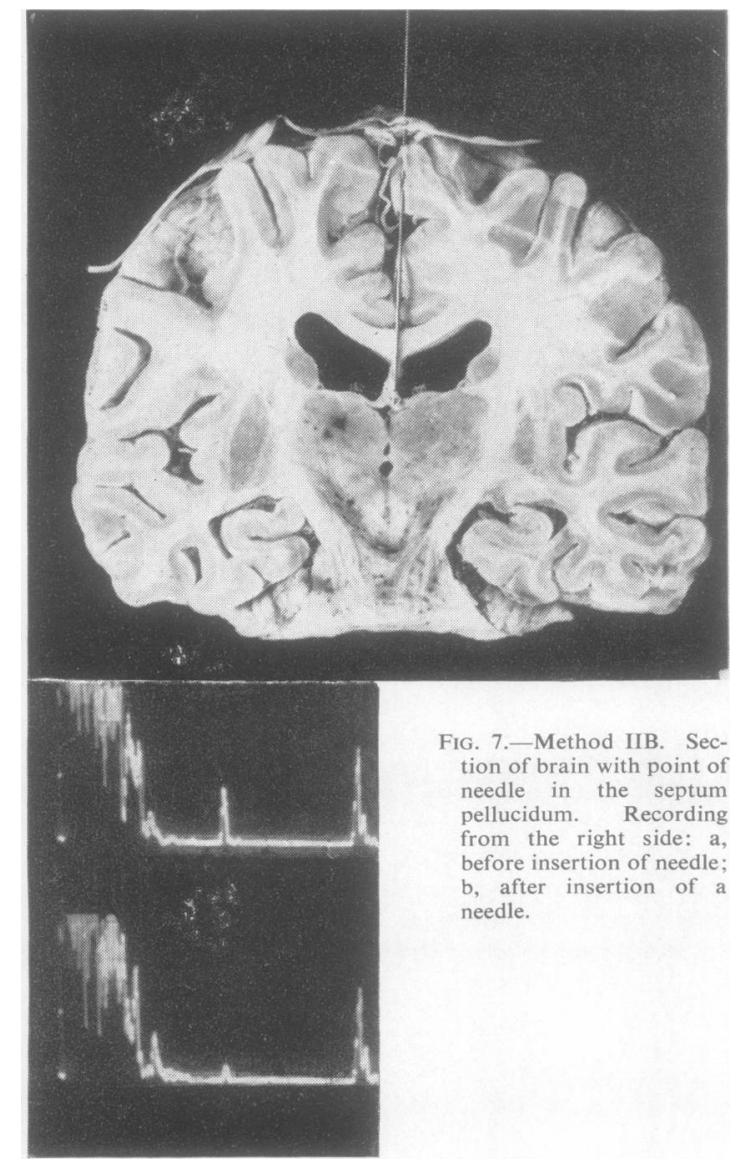

FIG. 7.-Method IIB. Section of brain with point of needle in the septum pellucidum. Recording from the right side: a, before insertion of needle; $b$, after insertion of a needle.

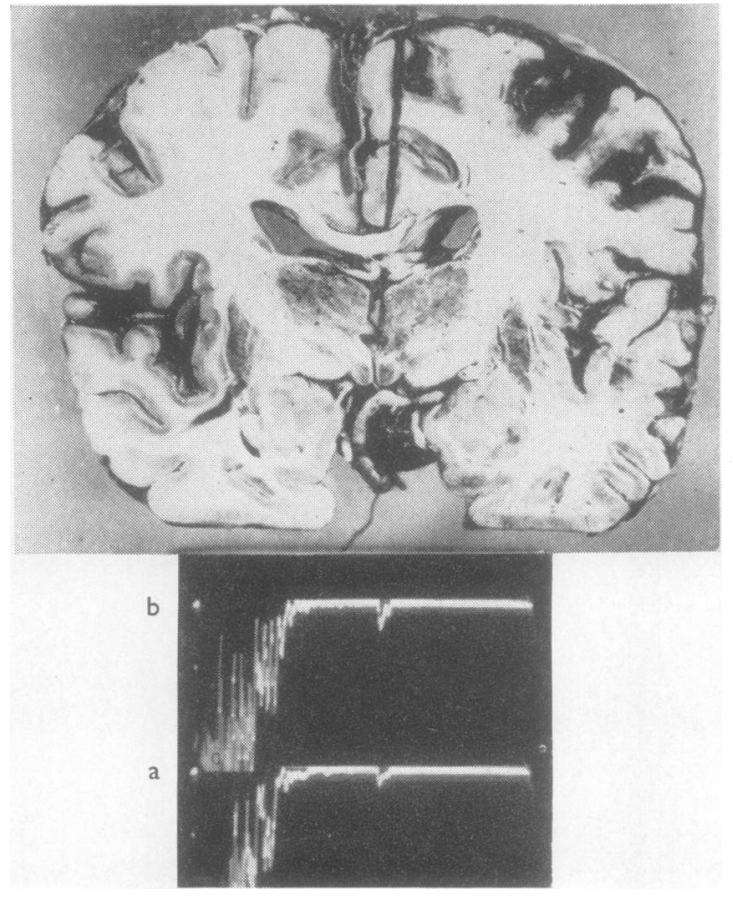

FIG. 9.-Method IIB. Section of brain with point of needle in the third ventricle: $a$, before insertion of needle; $b$, after insertion of needle.

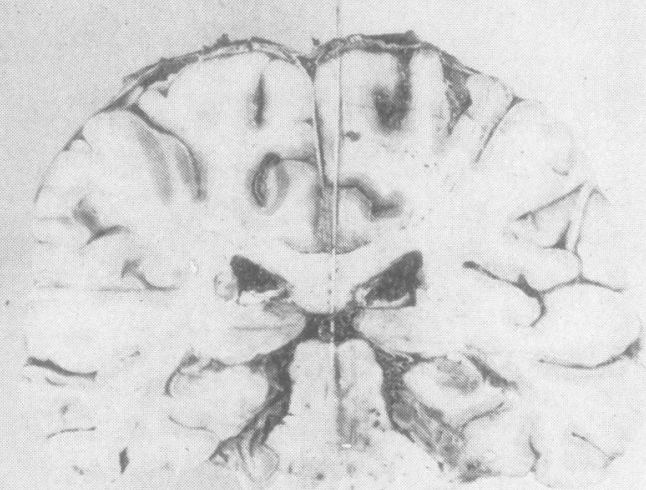

Fig. 8.-Method IIB. Section of brain with point of needle close to the aqueduct. Recording from the right side: a, before insertion of needle; $b$, after insertion of needle.

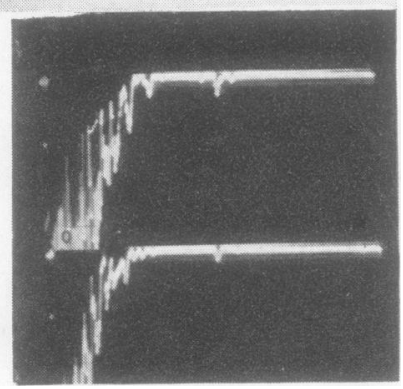

b

p q

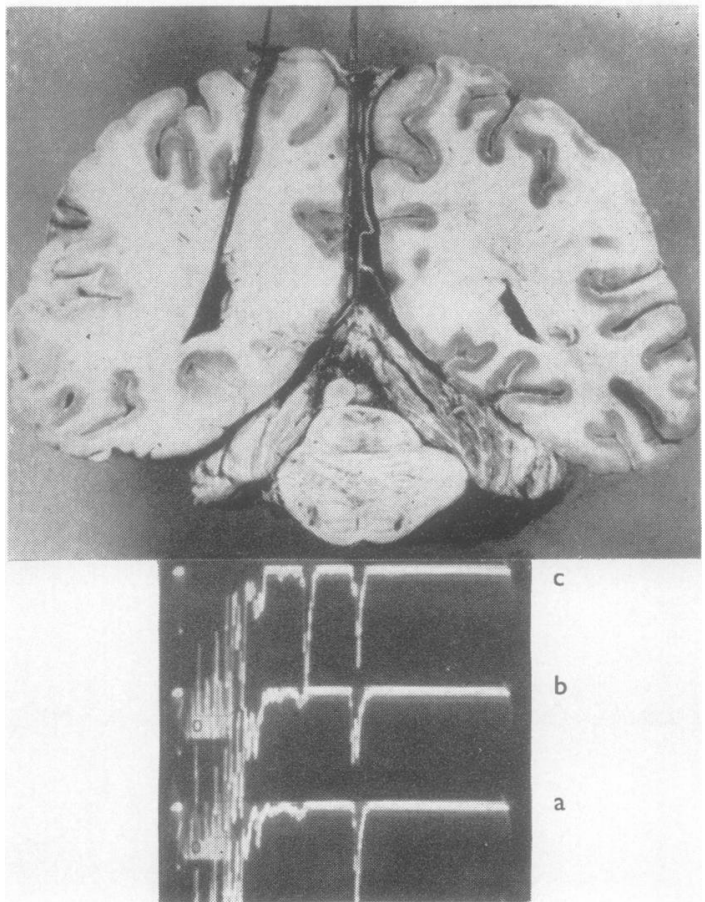

FIG. 10.-Method IIB. Section of brain with points of needles in the longitudinal fissure and in the left posterior horn. Recording from the left side: a, before insertion of needles; $b$, after insertion of needle $p$ : $c$, after insertion of needles $p, q$.

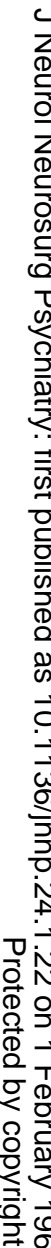


e

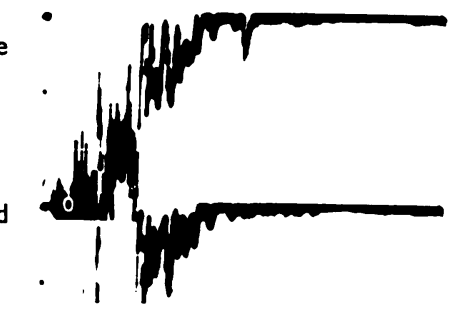

C

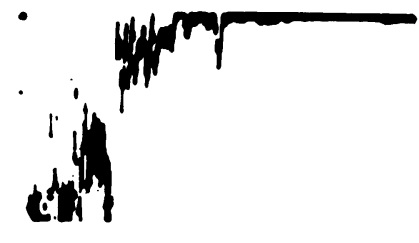

b

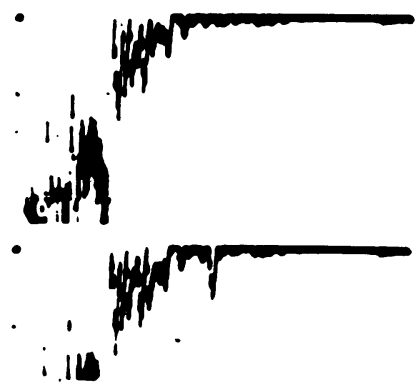

Fig. 11.-Echoes from falx under varying experimental conditions: a, echoencephalogram under normal conditions; b, after sectioning of falx; $c$, after stretching of falx; d, after removing falx; e, after replacing and stretching falx. foil with cellulose wadding between to trap air. In this way the probe is screened off with air so as to give a thin, slit-like beam of parallel ultrasonic rays along and immediately beneath the surface of the water.

Determination of the Horizontal Plane.-A brain is placed in a skull in order to reproduce as closely as possible the damping and refracting conditions of an investigation on the skull. The brain in the skull is placed in the water tank, and by moving the skull vertically the ultrasound can be made to pass into the brain in a clearly definable horizontal plane. Unprepared brains as well as brains fixed in formalin were used in the experiments. After it was found that the echoes arose in the same structures in both cases, brains fixed in formalin were commonly used for the sake of convenience.

Determination of the Frontal Plane.-The brain is os sectioned coronally and placed in the skull. The skull $\vec{\circ}$ is then immersed in the water-tank and moved in a sagittal direction until the beam just strikes the cut surface.

In this way the echo-producing structures on the cut $\stackrel{\leftrightarrow}{\stackrel{D}{\leftrightarrow}}$ surface of the brain can be determined. In doubtful cases and in order to check the dimensions of the ultrasonic beam, the needle is applied along the cut in surface next to the structure whose echo is to be analysed. The structure suspected to be the origin of the echo can i also be displaced to facilitate identification of the echo $N$ producing structures. By cutting off pieces of the brain 윽 successively, a complete echoencephalographic chart of the brain can be made.

\section{Results}

Fig. 12 illustrates two echoencephalogrant recorded at two different levels on the same sectiof of brain. The echoes in record a were recorded from the Sylvian fissure, the thalamus and the third

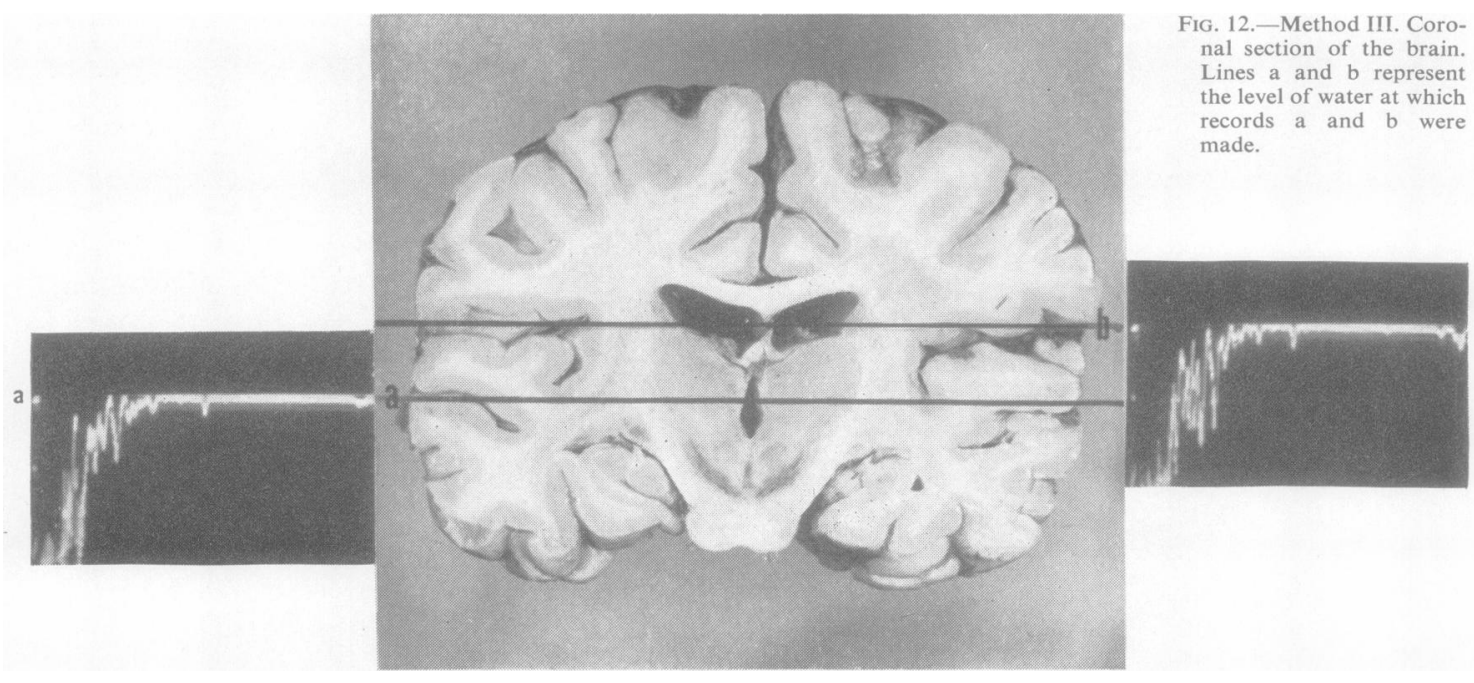




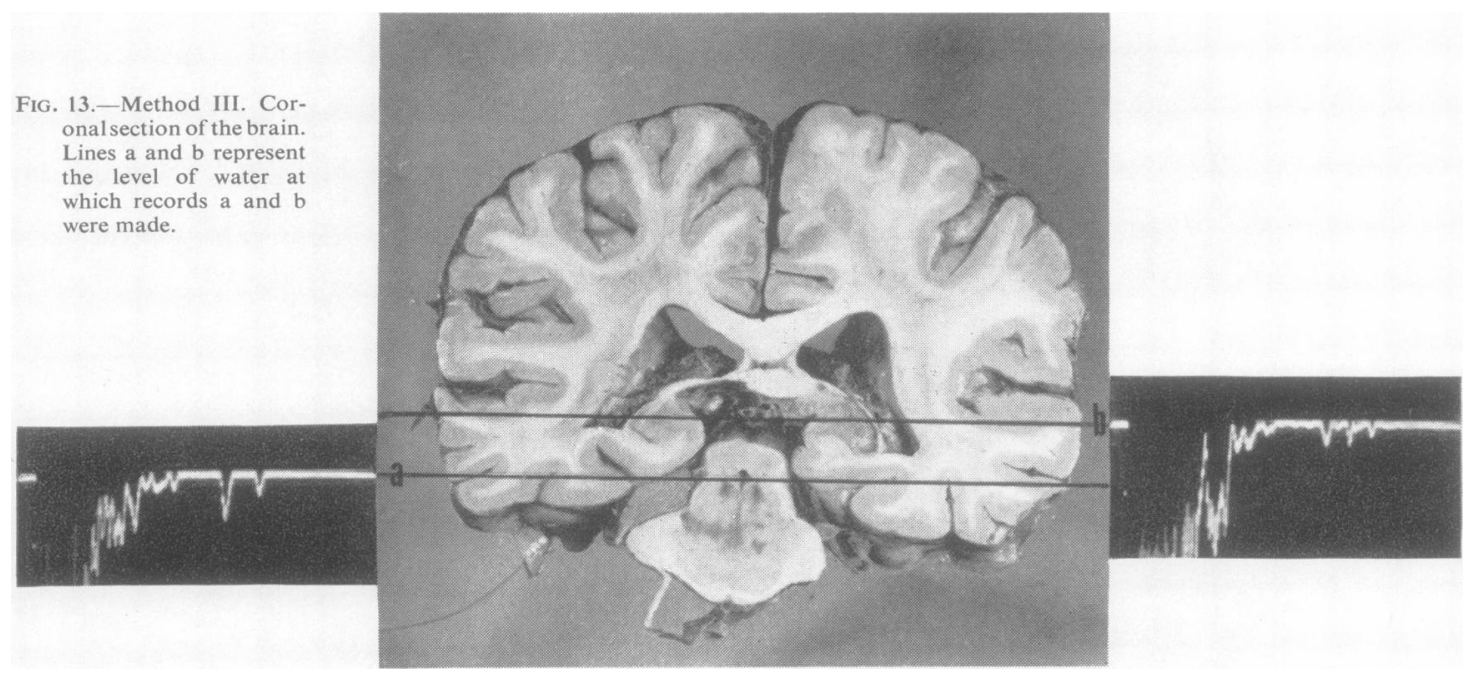

FIG. 14.-Method III. Coronal section of the brain. Lines $\mathrm{a}$ and $\mathrm{b}$ represent the level of water at which records $a$ and $b$ were made.
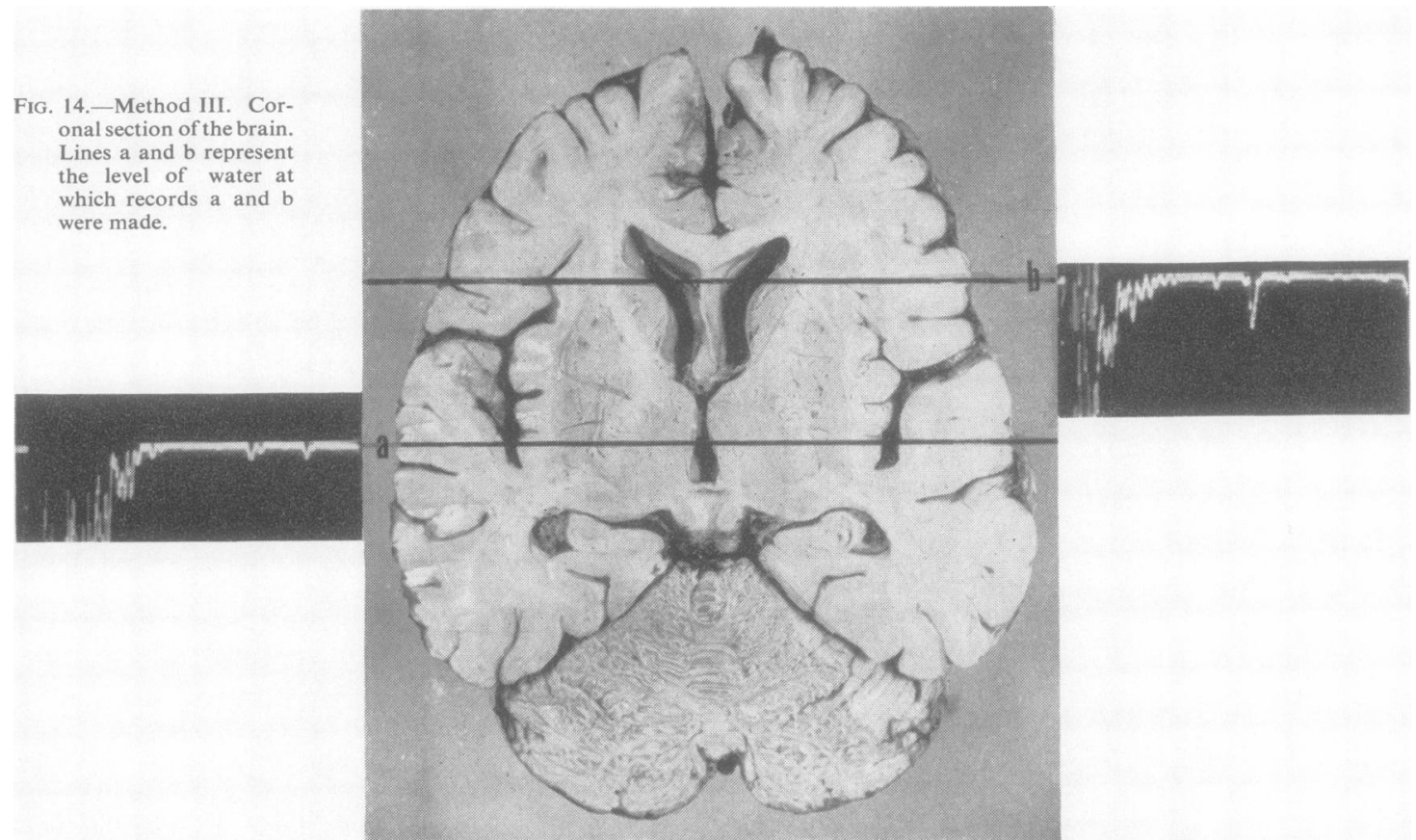

ventricle, in record $b$ from the Sylvian fissure, the lateral ventricle, and the septum pellucidum.

Fig. 13 illustrates two echoencephalograms recorded from a section of brain removed further occipitally. Record a has two distinct echoes in its central part; the left comes from the left lateral margin of the pons, while the right comes from the aqueduct.
In record $\mathrm{b}$, recorded in a somewhat higher horizontal plane, the echoes in the middle arise in the left margin of the transverse fissure, in the pineal body, and in the right margin of the transverse fissure.

Fig. 14 illustrates two echoencephalograms from a brain cut horizontally. The double midline echo in record a originates in the third ventricle and the 


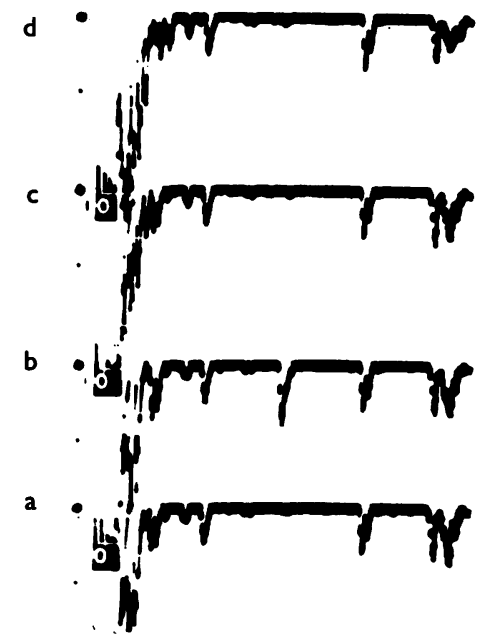

FIG. 15.-Echoencephalographic investigation of the pineal body through skull of normal thickness: a, record with pineal body removed; $b$, pineal body removed and point of needle shows its site; $c$, uncalcified pineal body in place; $d$, calcified pineal body in place.

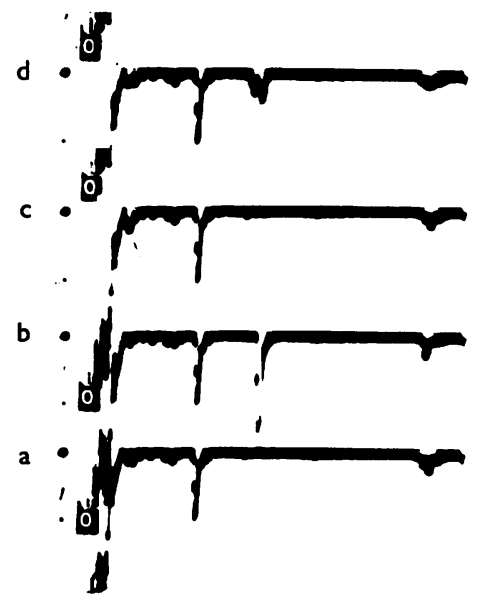

FIG. 16.-Echoencephalographic investigation of the pineal body through skull of exceptional thinness: $a$, pineal body removed; b, pineal body removed and point of needle shows its site; $c$, uncalcified pineal body in place; d, calcified pineal body in place. midline echo in record $b$ from the septum pellucidum.

Fig. 15 shows a special experiment with the pineal body.

A brain with a pineal body not visible on the radiograph was sectioned horizontally immediately 0 above the pineal body. The pineal was dissected and then replaced. The lower half of the brain with the pineal body was placed in a skull of normal thickness and then immersed in a tank of water.The experiment was performed both with a screened $\vec{F}$ probe and an unscreened probe.

Record a represents an echoencephalogram with the pineal body removed; no midline echo is seen. In record $b$ a needle has been held with the point at $\mathbb{D}$ the site of the pineal body to check that the ultra- $\frac{2}{0}$ sonic beam actually passed through the place where ${ }^{\infty}$ the pineal body would normally have been. $A \vec{O}$ strong midline echo was recorded. In record $c$ the $\overrightarrow{-}$ needle had been removed and the uncalcified pineal ${ }_{\sigma}$ body replaced; no midline echo was recorded. In record $d$, the uncalcified pineal body had beenreplaced by one which was visible on the radio-i graph; no midline echo was recorded here either. Thus no midline echo could be recorded throughin the skull from either an uncalcified or a calcified ${ }_{0}^{N}$ pineal body. Nevertheless midline echoes could ge recorded from, for example, the septum pellucidum $\vec{T}$ and the third ventricle.

Since the thickness of the bone of the skull might conceivably be the reason that no echo was recordex from the pineal body, the experiment was repeated in the same way with the same brains and with the same pineal bodies, but with an exceptionally thin. skull. Record a in Fig. 16 shows an echoencephalogram with the pineal body removed; no midline echo is seen. In record $b$ a needle echo was re-o corded from the former site of the pineal body. Record c shows an echoencephalogram with an uncalcified pineal body in place; no midline echo $\overrightarrow{\vec{P}}$ was recorded. In record $\mathrm{d}$ a double midline echo was 3 recorded from a calcified pineal body. Consequently an echo from a calcified pineal body can be recorded through the skull provided the bone is thin.

Using this water-tank method, the midline echo was recorded from the falx, the septum pellucidum, the third ventricle, the transverse fissure and the 3 aqueduct, and from a calcified pineal body if the skull was thin.

Lateral echoes arise from ventricles and gyri and from the boundary between gray and white substance.

\section{Discussion}

The purpose of the investigation was primarily to determine which structures in the brain give echoes 
recordable on the skull. With the exception of the experiments using method IIA, the recordings were made through the skull.

It appeared from all the experiments that the midline echo is recorded from different structures. Which structure gives an echo seems to depend primarily on the placing of the probe and to some extent also on the shape and thickness of the skull.

A summary of the results of the investigation indicates that midline echoes may be formed by the following structures: aqueduct, transverse fissure, pineal body, third ventricle, septum pellucidum, and falx cerebri.

According to Leksell, the midline echo is produced primarily by the pineal body, whether calcified or not. He bases this opinion on experiments on brains in a tank of water without having the ultrasound pass through the skull. But it is clear from my investigation that a pineal body which cannot be visualized radiologically gives no echo which can be recorded through the skull. On the other hand, a pineal body with visible calcification can be recorded on the echoencephalogram through the skull on the condition that the bone is relatively thin. If the skull is relatively thick or dense, the damping effect will be so great that the echo of the pineal body cannot be recorded even though other midline echoes can be recorded, e.g., from the falx cerebri, the septum pellucidum, and the third ventricle. The fact that echoes can be recorded from these structures when the pineal body does not give an echo is probably due to their having plane surfaces which effectively reflect the ultrasound, whereas the pineal body is a round structure against whose surface the ultrasound is reflected in different directions.

According to Vlieger and Ridder, the longitudinal fissure, but not the falx, is the source of the midline echo. They base this view on their findings that 'on several occasions a double midline reflection was seen'. By placing a thin steel blade in the longitudinal fissure, Vlieger and Ridder found that 'the reflection of the steel blade appeared exactly between the double midline reflection'. It would appear that the probe used was a $1 \mathrm{Mc}$./sec. with a diameter of $34 \mathrm{~mm}$. If so, the ultrasonic ray would be approximately $34 \mathrm{~mm}$. in diameter, and it would then be impossible to specify which structures in the midline yielded the echo. Even if one makes the recording high on the aural vertical, one cannot be sure with this probe that the midline echo does not come from the third ventricle, for example. An echo from the third ventricle is usually recorded as a double echo of the type Vlieger and Ridder demonstrated to prove their claim. That a thin steel blade in the longitudinal fissure gives an echo recorded in the middle of the double echo does not prove that the double echo arises in the longitudinal fissure. On the other hand, their opinion that the falx cerebri does not yield a distinct echo is disproved by the present investigation.

With regard to the lateral echoes, earlier researchers have considered that the ventricular walls give echoes. This view is based on the location of the lateral echoes on the echoencephalogram, although no method of verifying it has been presented. With the methods described above, however, it was possible to prove that this view is correct. Furthermore, it was found that echoes originate in the gyri, particularly the Sylvian fissure.

The finding that the boundary between gray and white substance yields an echo is somewhat surprising and is of essential interest. From the boundary between the thalamus and the internal capsule echoes can be recorded, which may easily be confused with ventricular echoes.

\section{Summary}

It was found that midline echoes could be recorded in infants over a lateral area which is relatively much larger than in adults. This suggests that the midline echo is not only produced by so small a body as the pineal.

A method using needle indicators in order to determine which structures give echoes is described. These experiments were performed on cadavers, both directly on the brain and on the skull.

The location of the echoes can be determined with greater precision with a screened ultrasonic beam in a tank of water by successive removal of parts of the brain.

These investigations show that midline echoes can be recorded from the falx cerebri, the septum pellucidum, the third ventricle, the transverse fissure, the pineal body, and the aqueduct. Lateral echoes originate in a number of boundary surfaces between brain and cerebrospinal fluid (ventricles and gyri) and, under favourable conditions, can be recorded from the boundary between gray and white substance (thalamus, internal capsule, and external capsule).

I wish to thank Prof. L. Leksell and Dr. S. Jeppson, Lund, who have kindly instructed me in the use of the Krautkrämer's apparatus. I am also indebted to $\mathbf{M r}$. S. Dahn, Technical X-ray Centre, Stockholm, for valuable advice. This study has been supported in part by the Association for the Aid of Crippled Children, New York.

\section{REFERENCES}

Gordon, D. (1959). Brit. med. J., 1, 1500.

Jeppson, S. (1960). Personal communication.

Leksell, L. (1956). Acta chir.

Lithander, B, (1960a). Acta. psychiat. scand., 35, 235.

- (1960b). Ibid., 35, 241.

Vlieger, M. de, and Ridder, H. J. (1959). Neurology, 9, 216. 